\title{
Intra osseous dermoid cyst of mandible-A rare case report
}

\author{
Vibha Singh $^{1^{*}}$, Shalini Gupta ${ }^{2}$, Ridhi Jaiswal ${ }^{3}$, Laxman Malkunje ${ }^{1}$ \\ ${ }^{1}$ Department of Oral and Maxillofacial Surgery, Chhatrapati Shahuji Maharaj Medical University, Lucknow, India \\ ${ }^{2}$ Department of Oral Pathology, Chhatrapati Shahuji Maharaj Medical University, Lucknow, India \\ ${ }^{3}$ Department of Pathology, Chhatrapati Shahuji Maharaj Medical University, Lucknow, India \\ Email: *
}

Received 21 March 2012; revised 25 April 2012; accepted 5 May 2012

\begin{abstract}
The present article report a rare case of intraosseous dermoid cyst in mandible which clinically and radiographicaly presented as mandibular cyst. The diagnosis of dermoid cyst was confirmed histologically. A 20 year old patient was admitted to our Department with the complaint of swelling in left lower jaw with occasional pain. Radiographic examination revealed a radiolucent lesion extending from left $2^{\text {nd }}$ molar to right premolar region. On surgical exploration the lesion was cystic in nature full of hair and keratinized tissues. The tissue was sent for the histopathological examination and macroscopic feature reveal numerous bits of soft tissue. The tissues were creamish brown in color, firm in consistency, with largest tissue measuring $2.5 \times 1.5 \times 0.5 \mathrm{~cm}$ in dimension. Few hair follicles were also evident within it. The histopathology reveal cyst lined by stratified squamous hyperorthokeratinized epithelium supported by a fibrous connective tissue wall. The cystic epithelium is showing melanin pigmentation in the basal cell layer. Abundant onion skin keratin is seen within the cyst lumen. The epithelial lining as well as cyst wall shows numerous skin appendages such as lobules of sebaceous glands. The underlying connective tissue shows mild chronic inflammatory infiltrate mainly composed of lymphocytes.
\end{abstract}

Keywords: Dermoid Cyst; Dermal Appendages; Epidermoid Cyst; Teratoma

\section{INTRODUCTION}

Epidermoid and dermoid cysts are developmental anomalies. They are basically cystic teratomas, resulting primarily from the trapped germinal epithelium. They occur in all parts of the body but, rarely in the oral cavity. The floor of the mouth is the most common site in oral cavity, followed by submandibular and sublingual region. Intra

*Corresponding author. osseous epidermal cyst and dermoid cyst is extremely rare in the maxillofacial region. Only 13 cases have been documented in the literature, 7 in mandible and 6 in maxilla [1].

\section{CASE REPORT}

A 20 year old male reported to the Department of Oral and Maxillofacial Surgery with a complaint of swelling in the left lower jaw for one year.

On intra oral examination, a solitary swelling extending from left $3^{\text {rd }}$ molar (38) to right $1^{\text {st }}$ premolar (44) was seen. The overlying mucosa was normal and intact. On palpation the swelling was non tender and firm in consistency with intact cortical plates. On further investigation, the patient did not reveal any other complaint such as disturbance of sensation in the distribution area of mental and mandibular nerve. Lymph nodes were not palpable.

An Orthopantomogram was advised, which revealed a radiolucent lesion extending from the medial root of left third molar up to right first premolar region with resorption of the roots (Figure 1). CT scan showed radiolucent lesion in left body of the mandible (Figures 2 and 3). Based on these findings the clinical differential diagnosis of Odontogenic Keratocyst, Ameloblastoma and Central giant cell granuloma was made. Fine Needle Aspiration Cytology was performed which showed small clusters of keratin squames, based on this the cytological differential diagnosis of Odontogenic Keratocyst was made.

On surgical exploration under general anaesthesia, revealed cystic cavity containing hair follicles, so the other differential diagnosis of dermoid cyst was made. The enucleation of lesion was performed (Figures 4 and 5). Post operative healing was uneventful.

\section{HISTOLOPATHOLOGY}

The tissue was sent for the histopathological examination and macroscopic feature reveal numerous bits of soft tissue. The tissues were creamish brown in color, firm in consistency, with largest tissue measuring $2.5 \times 1.5 \times 0.5$ 


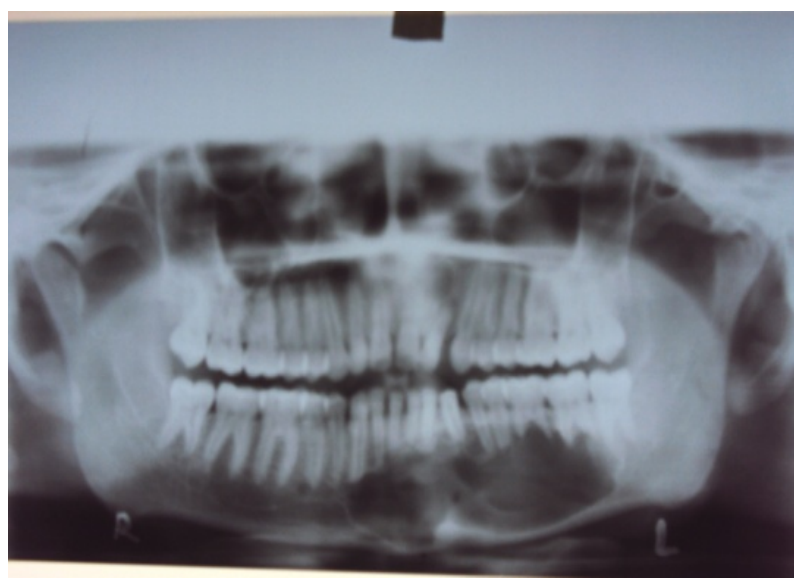

Figure 1. X-ray OPG of jaws showing radiolucent lesion extending from left molar to right premolar region.

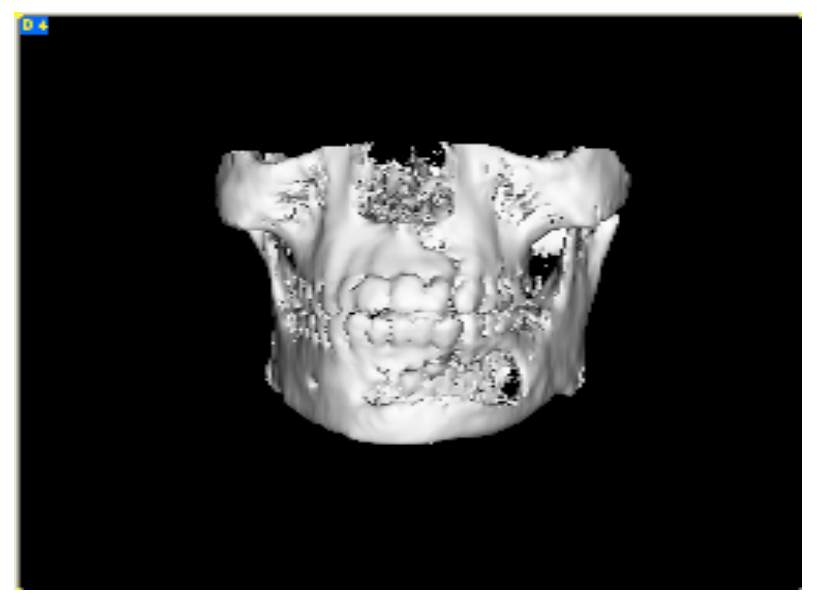

Figure 2. CT scan 3-D reconstruction showing lesion on left body of the mandible.

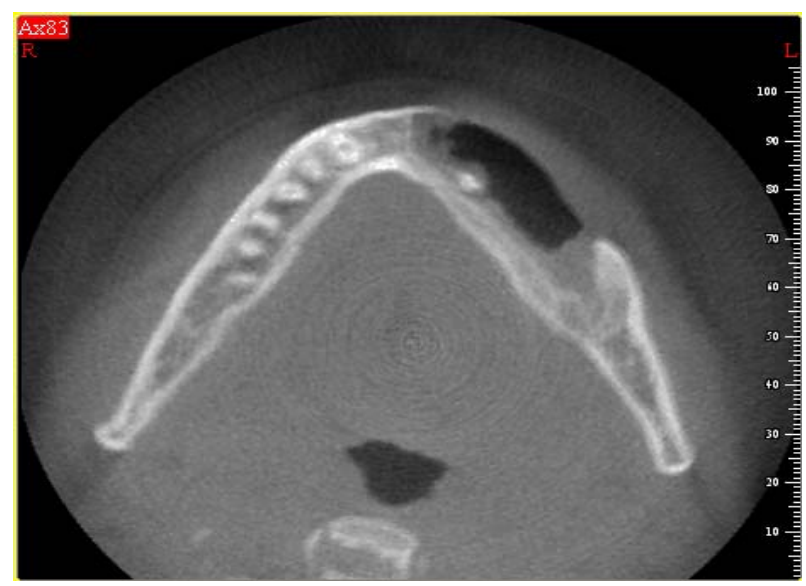

Figure 3. CT scans axial view showing radiolucent lesion with intact lingual cortical plate.

$\mathrm{cm}$ in dimension. Few hair follicles were also evident within it (Figure 6). The histopathology reveal cyst lined by stratified squamous hyperorthokeratinized epithelium supported by a fibrous connective tissue wall. The cystic

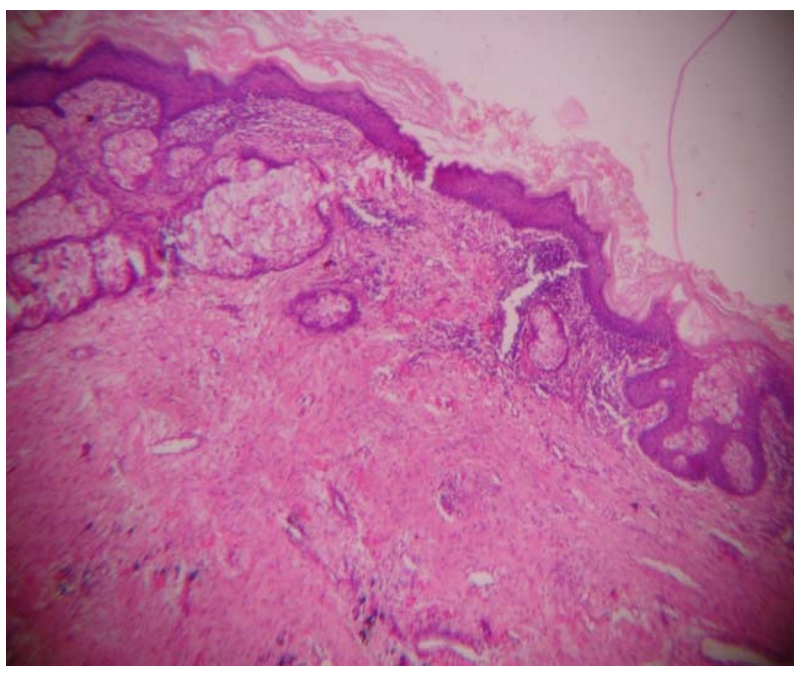

Figure 4. Intra operative photograph showing cystic cavity with few hair.

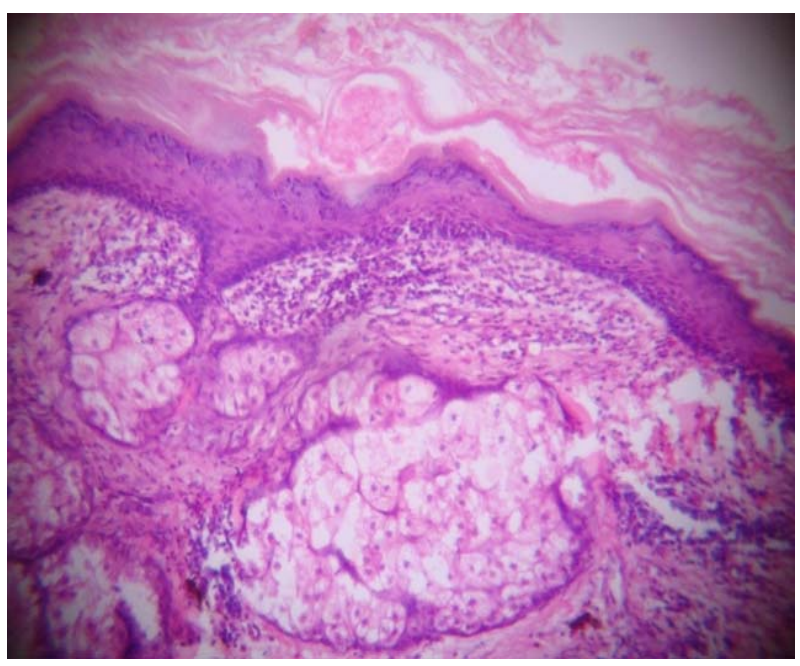

Figure 5. Tissue specimen with hair removed immediately after surgery.

epithelium is showing melanin pigmentation in the basal cell layer. Abundant onion skin keratin is seen within the cyst lumen. The epithelial lining as well as cyst wall shows numerous skin appendages such as lobules of sebaceous glands. The underlying connective tissue shows mild chronic inflammatory infiltrate mainly composed of lymphocytes (Figures $\mathbf{7}$ and $\mathbf{8}$ ).

\section{DISCUSSION}

A search of the English-language literature 1966 to 2009 yielded 13 cases of true intra osseous dermoid cyst of the jaws. Most commonly seen in age range of 5 to 69 years (mean 32) and a M:F ratio of 1:1.5. Six cases occurred in the maxilla, most frequently in hard palate and incisor canine area, 8 in the mandible, most frequently in the molar area. 


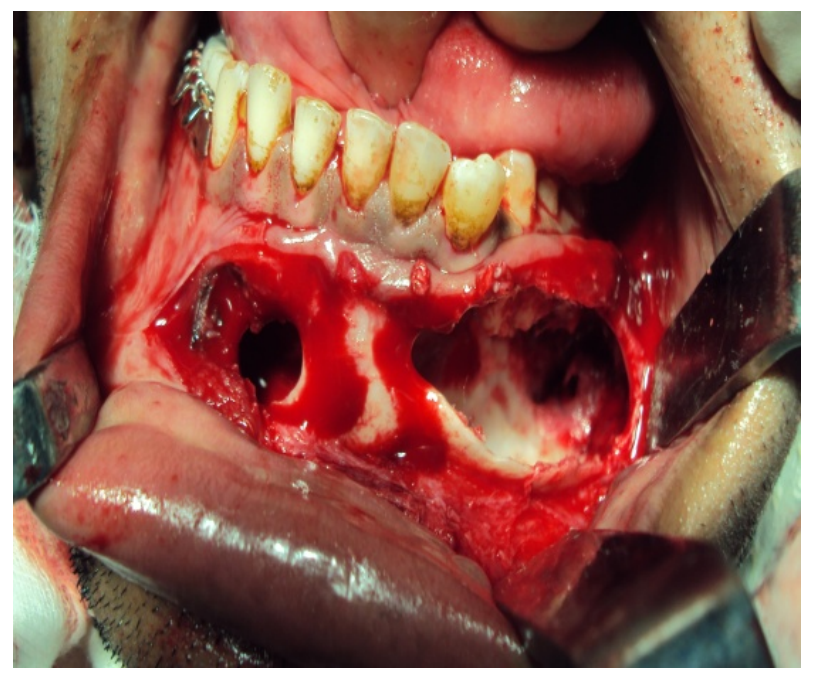

Figure 6. Gross specimen photograph showing tissue specimen with hair (Formalin fix).

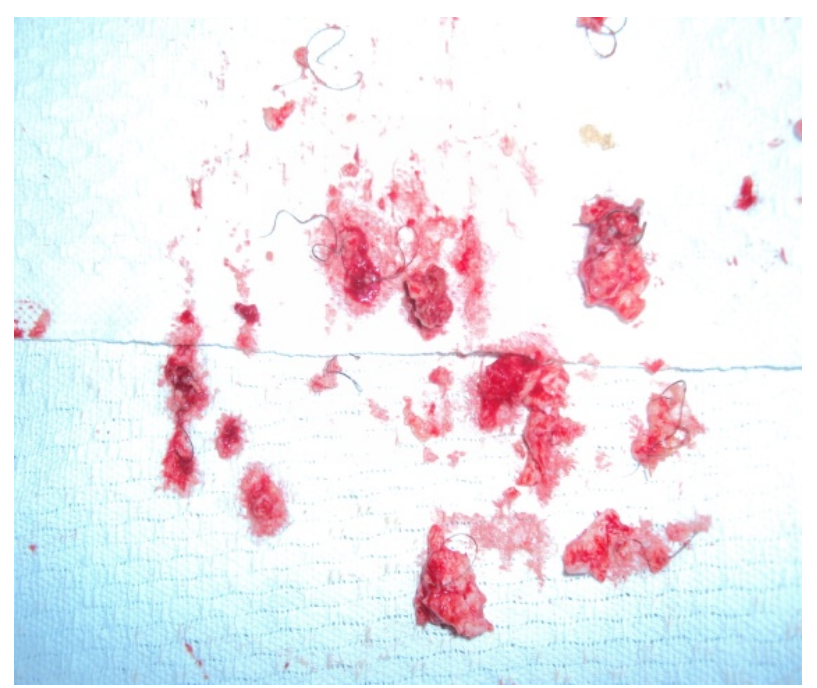

Figure 7. Photograph under $4 \times$ magnification showing cystic epithelium with onion skin keratin in the lumen and the skin appendages like sebaceous gland within epithelium and cystic wall.

The term dermoid cyst is used to describe three closely related cystic lesions, epidermoid cyst, dermoid cyst and teratoma. The epidermoid cyst (simple type) is characterized by a stratified keratinized epithelial lining, resembling epidermis, devoid of skin appendages such as, hair follicles, sebaceous cyst or sweat glands. The dermoid cyst (compound type) is defined similarly as that of the epidermoid type but contains skin appendages such as hair follicles, sebaceous glands or sweat glands. Teratomas (complex type) includes tissues of both mesodermal and endodermal origin such as cartilage, muscles and nerves [2]. Pathogenesis of such cysts have always been controversial dermoid cyst usually occur in situations where coalescence takes place between cutaneous

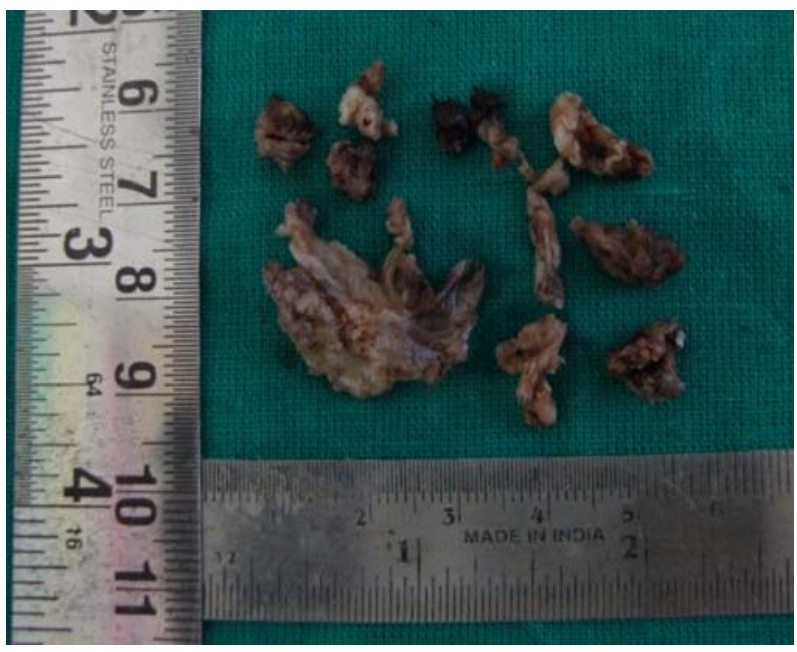

Figure 8. Photograph under $10 \times$ magnification showing stratified squamous hyper-orthokeratinized epithelium with sebaceous gland.

surfaces. These of the face are not uncommon, but are most frequently found in connexions with the nose, orbit or cheek [3].

Hence we report an unusual rare case of intraosseous cystic lesion, that developed in mandible of a 20 years old male. It is important to differentiate between keratocyst and intra osseous dermoid cyst ,apparently slow growth together with absence of recurrence in case indicate an indolent biological behavior for dermoid cyst, as opposed to OKC which is an aggressive lesion with a high recurrence rate.

Numerous authors have given reasons for such uncommon occurrences including de novo origin, or could be sequestration of stomadeal ectoderm that may have been trapped deep in the surface and embedded in the developing mandibular bone during embryogenesis [1, $3-5]$. We suggest that in our case the most possible reason could be embryonic epithelium migration.

Histologically both dermoid and epidermoid cysts are lined by keratinized stratified squamous epithelium resembling epidermis. Occasional cases may have areas of pseudostratified ciliated columnar epithelium but cysts of the floor of the mouth lined predominantly by secretory epithelium are probably of salivary duct origin. The dermoid cyst is characterized by the presence in the wall of one or more dermal appendages such as hair follicles, sweat glands or sebaceous glands. Hair is very rarely found. The lumen is usually filled with Keratin.

The dermoid cyst is a form of cystic teratoma derived principally from embryonic germinal epithelium, but in some instances it also may contain structures of the other germ layers. Some authors use the term dermoid cyst as a synonym for Teratoma meaning any Teratoma regardless of its histology and locations [2].

New and Erich reported a series of 103 dermoid cysts 
of head and neck region the most common sites are in the soft tissue of the head and neck region and noted that the floor of the mouth and submaxillary and sublingual areas were considered to be most common site of occurrence [6,7].

Dermoid cysts in the mandible are considered to be derived from enclavement of epithelial debris in the midline during closure of the mandibular and hyoid branchial arches. Since some of these cells are totipotent blastomeres, the occurrence of non-epithelial structures can be readily understood [8].

\section{SUMMARY AND CONCLUSION}

The intra osseous dermoid cyst is a rare non aggressive lesion. It shares some features with odontogenic keratocyst but hair follicles, sebaceous glands are present only in the dermoid cyst. Incomplete excision may lead to recurrence of cyst. The case reported above depicts a rare occurrence of intra osseous mandibular cyst but on histopathological examination revealed dermal appendages leading to diagnosis of dermoid cyst.

\section{REFERENCES}

[1] Dror, M., Allon, S.C. and Ilana, K. (2010) Intraosseous com- pound type dermoid cyst of the jaw. International
Journal of Head and Neck Surgery, 1, 103-106.

[2] Bonder, L., Woldenberg, Y. and Sino, V.N. (2005) Dermoid cyst of maxilla. International Journal of Head and Neck Surgery, 34, 453-455

[3] Komiyama, K., Miki, Y., Oda, Y., Tachibana, T., Okaue, M., Tanaka, H. and Moro, I. (2002) Uncommon dermoid cyst presented in the mandible possibly originating from embryonic epithelium remnants. Journal of Oral Pathology \& Medicine, 31, 184-187. doi:10.1034/j.1600-0714.2002.310311.x

[4] Wood, N.K. and Goaz, P.W. (1998) Differential diagnosis of oral and maxillofacial lesions. 5th Edition, Indian Edition, Noida.

[5] Craig, G.T., Holland, C.S. and Hindle, M.O. (1980) Dermoid cyst of the mandible. British Journal of Oral Surgery, 18, 230-237. doi:10.1016/0007-117X(80)90067-0

[6] Russell, E., Christensen, Jr. and Robert, H. (1982) Intra osseous mandibular cyst with sebaceous differentiation. Oral Surgery, Oral Medicine, Oral Pathology, 53, 591595. doi:10.1016/0030-4220(82)90346-2

[7] Shafer, W.G, Hine, M.K. and Levy, B.M. (1983) A text book of oral pathology. 4th Edition, W. B. Saunders Company, Philadelphia.

[8] Takeda, Y., Oikawa, Y., Satoh, M. and Nakamura, S. (2003) Latent form of multiple dermoid cyst in the jaw bone. Pathology International, 53, 786-789. doi:10.1046/j.1440-1827.2003.01560.x 